\title{
Valuations revisited
}

\author{
M. P. Schellekens
}

\begin{abstract}
The notion of a semivaluation has been introduced in [Sch98]. Aside from providing a novel concept generalizing valuations on lattices to the context of semilattices, semivaluations shed new light on the notion of a "partial metric" well known from theoretical computer science (e.g. [Mat94], [Mat95], [O’N97], [BS97] and [BSh97]).

As discussed in [Sch98], the characterization of partial metrics in terms of semivaluations is non-trivial and involves the solution of an open problem of the Survey Paper "Non-symmetric Topology" (Problem 7 of [Kün93]) for the class of quasi-uniform semilattices.

We recall from [Sch98] that the traditional domain theoretic examples, including the well known class of totally bounded Scott domains (e.g. [Smy91]), all correspond to semivaluation spaces. As such it is possible to study Quantitative Domain Theory (e.g. [FSW96]) in this simplified context, similar to the study of metric lattices (uniform lattices) in the more basic context of valuation spaces (cf. [Bir84] and also [Web91]). Hence the notion of a semivaluation is of sufficient interest to merit an independent study.

The main purpose of this short note is to provide a basic introduction to the notion of a semivaluation independent of the domain theoretic considerations of [Sch98] and to discuss a recently obtained characterization of valuations in terms of semivaluations.
\end{abstract}

\section{Introduction}

Valuations have been studied extensively in the literature. We recall from Birkhoff's well known book on Lattice Theory ([Bir84]), that: "many of the most important applications of lattices to mathematics involve limiting processes like those of real analysis. Such processes can be defined in many ways, ... The simplest way is in terms of "valuations", ..." .

We recall that valuations also have been used in Theoretical Computer Science (where they sometimes are referred to as "evaluations"). Valuations are fundamental notions in the development of a probabilistic powerdomain (e.g. [JP89] and [Jon89]) as well as in connection to the development of a domain theoretic treatment of integration (e.g. [Eda94]) and in the context of the development of a lower bag domain as a semantics for nondeterministic programs ([Hec95]).

Recent developments in Domain theory indicate that additional concepts are required in order to develop the corresponding applications. These developments include domain theoretic approaches to dataflow networks (e.g. [Mat94] and [Mat95]), logic programming (e.g. [Sed96]), computational models for the reals (e.g. [Eda94]), 
probabilistic languages (e.g. [Jon89] and [JP89]), as well as models which incorporate complexity analysis (e.g. [Sch96] and [RS96]).

Each of these applications involves "real number measurements" in some sense, and hence the terminology quantitative as opposed to the traditional qualitative (= order theoretic) approach.

The question remains as to how "quantities" can be introduced to classical Domain Theory in a simple and elegant way. A main theme of [Sch98] is the introduction of the novel concept of a semivaluation on a semilattice. It follows from [Sch98] that this notion provides a minimal extension of traditional domain theory, sufficient to develop the above mentioned applications.

The semivaluation approach thus has the advantage that it allows for a uniform presentation of the traditional quantitative domain theoretic structures and applications, as for instance the totally bounded Scott Domains of [Smy91], the partial metric spaces of [Mat95] and the complexity spaces of [Sch95]. The notion allows one in addition to incorporate the above mentioned applications of theoretical computer science based on the classical notion of a valuation, in the more general context of the theory of semivaluations.

In this sense, the key result provided in this paper (corollary 7) is fundamental, as it shows that valuations on a lattice naturally consist of a combination of the dual notions of a join valuation on a join semilattice and of a meet valuation on a meet semilattice.

As such the generalized notion of a semivaluation on a semilattice not only allows one to present most of the recent domain theoretic applications involving real number measurements in a unified way, but it also provides a natural generalization of the traditional notion of a valuation on a lattice to the context of semilattices.

\section{Background}

A join (meet) semilattice is a partial order $(X, \leq)$ such that every two elements $x, y \in X$ have a supremum $x \sqcup y$ (infimum $x \sqcap y$ ) in $X$. A lattice is a partial order which is both a join and a meet semilattice.

We recall the definition of a valuation on a lattice $(L, \sqsubseteq)$.

Definition 1 A function $f: L \rightarrow \mathcal{R}_{0}^{+}$is a valuation iff

(1) $f$ is increasing, i.e. $\forall x, y \in L . x \sqsubseteq y \Rightarrow f(x) \leq f(y)$.

(2) $\forall x, y \in L . f(x \sqcap y)+f(x \sqcup y)=f(x)+f(y)$.

Examples: The cardinality function on a powerset $P(A)$ of a set $A$, ordered by subset inclusion, is a valuation. Probability measures are valuations. 
In case the function $f$ is decreasing and satisfies (2), we refer to $f$ as a covaluation.

If $f$ only satisfies (2) we say that $f$ satisfies the modularity law, or also that $f$ is modular.

There does not seem to be a consistent terminology in the literature. Valuations, also called evaluations, as used in computer science (e.g. [BS97] or [Jon89]) typically satisfy (1) and (2). In the classical mathematical literature a valuation only needs to satisfy (2) (e.g. [Bir84]).

It is convenient for matters of presentation to reserve the definition given above for a valuation in order to state results on valuations as they occur in Computer Science.

Definition $2 A$ (co)valuation $f$ on a lattice $(L, \sqsubseteq)$ is called positive (negative) if $f$ is strictly increasing (decreasing), i.e. $\forall x, y \in L . x \sqsubset y \Rightarrow f(x)<f(y)(f(x)>f(y))$.

In using the terminology "positive" we follow the classical terminology of [Bir84]. We remark that the positiveness requirement corresponds to the strongly non-degenerate requirement of [BSh97] and that positive valuations are exactly the dimension functions as defined in [CD73].

Definition 3 If $(X, \preceq)$ is a join semilattice then a function $f:(X, \preceq) \rightarrow \mathcal{R}_{0}^{+}$is joinmodular iff

$$
\forall x, y, z \in X . f(y \sqcup z)-f(x \sqcup z) \geq f(y)-f(x \sqcup y)
$$

and $f$ is co-join-modular iff

$$
\forall x, y, z \in X . f(y \sqcup z)-f(x \sqcup z) \leq f(y)-f(x \sqcup y)
$$

A join valuation on a join semilattice is a join-modular increasing function on this semilattice. A join co-valuation on a join semilattice is a co-join-modular decreasing function on this semilattice. The terminology of "positive" and "negative" is used in this context similarly to the convention for (co-)valuations.

Definition 4 If $(X, \preceq)$ is a meet semilattice then a function $f:(X, \preceq) \rightarrow \mathcal{R}_{0}^{+}$is meet-modular iff

$$
\forall x, y, z \in X . f(y \sqcap z)-f(x \sqcap z) \leq f(y)-f(x \sqcap y)
$$

and $f$ is co-meet-modular iff

$$
\forall x, y, z \in X . f(y \sqcap z)-f(x \sqcap z) \geq f(y)-f(x \sqcap y)
$$

A meet valuation on a meet semilattice is a meet-modular increasing function on this semilattice. A meet co-valuation on a meet semilattice is a co-meet-modular decreasing function on this semilattice. The terminology "positive" and "negative" is used in this context similarly to the convention for (co-)valuations. 
Some additional insight into these notions can be gained from the fact that the inequality for join modularity, i.e. $f(y \sqcup z)-f(x \sqcup z) \geq f(y)-f(x \sqcup y)$, is equivalent to $f(x \sqcup z) \leq f(x \sqcup y)+f(y \sqcup z)-f(y)$, which reflects the triangle inequality for partial metrics.

Indeed, partial metrics, as introduced by Matthews in [Mat94], form a generalization of ordinary metrics where the distance between a point and itself need not be zero. Hence the triangle inequality for a partial metric $p$ is formulated as: $p(x, z) \leq p(x, y)+p(y, z)-p(y, y)$, where the term $p(y, y)$ is substracted to compensate for this fact.

Since the work in [Sch98] shows there is a tight relationship between such partial metric "weighting functions" $p(y, y)$ on a join semilattice and join-modular functions, it is not surprising that the law for join-modularity takes this shape. A similar remark holds for co-meet-modulartiy.

The laws of co-join-modularity and meet-modularity simply express the opposite inequalities and mainly play a role in expressing dual results (cf. [Sch98]).

Definition 5 A function is a semivaluation if it is either a join valuation or a meet valuation. A join (meet) valuation space is a join (meet) semilattice equipped with a join (meet) valuation. A semivaluation space is a semilattice equipped with a semivaluation.

Example: Let $(X, \leq)$ be a meet semilattice, $A$ a countable subset of $X$ and let $w: X \rightarrow \mathcal{R}_{0}^{+}$be a function such that $\sum_{a \in A} w(a)<\infty$. The function $\bar{w}$ is then generated from $w$ on $X$ as follows:

$$
\forall x \in X . \bar{w}(x)=\sum_{a \in A}\{w(a) \mid a \in x \downarrow\}
$$

where $x \downarrow=\{a \in A \mid a \leq x\}$. One can easily verify that $\bar{w}$ is a meet valuation on $(X$, $\leq)$

\section{The characterization}

Proposition 6 An increasing function $f: L \rightarrow \mathcal{R}_{0}^{+}$on a lattice $L$ is modular iff it is join-modular and meet-modular. A decreasing function $f: L \rightarrow \mathcal{R}_{0}^{+}$on a lattice $L$ is modular iff it is co-join-modular and co-meet-modular.

Proof: We present the proof for the case of decreasing functions. The proof for the case of increasing functions is similar.

Let $L$ be a lattice and let $f$ be a decreasing modular function on $L$.

We have that $\forall x, y, z \in X . f(y \sqcup z)-f(x \sqcup z) \leq f(y \sqcup z)-f(x \sqcup y \sqcup z)$ since $f$ is decreasing. 
So it suffices to verify that $f(y \sqcup z)-f(x \sqcup y \sqcup z) \leq f(y)-f(x \sqcup y)$. For this it suffices to verify that

$$
(*) a \geq b \Rightarrow f(a)-f(a \sqcup c) \leq f(b)-f(b \sqcup c) .
$$

Since $f$ is modular, we have that $f(a \sqcup c)=f(a)+f(c)-f(a \sqcap c)$ and $f(b \sqcup c)=$ $f(b)+f(c)-f(b \sqcap c)$.

So we obtain that $(*)$ is equivalent to the statement

$$
a \geq b \Rightarrow f(a \sqcap c) \leq f(b \sqcap c) .
$$

This last inequality follows by the fact that $f$ is decreasing. Hence $f$ is co-joinmodular.

By the modularity of $f$ this is equivalent to $\forall x, y, z \in X . f(y)+f(z)-f(y \sqcap z)-$ $[f(x)+f(z)-f(x \sqcap z)] \leq f(y)-[f(x)+f(y)-f(x \sqcap y)]$, which is equivalent to $\forall x, y, z \in X . f(y \sqcap z)-f(x \sqcap z) \geq f(y)-f(x \sqcap y)$. Hence $f$ is co-meet-modular.

To show the converse, it suffices to verify that any function $f: L \rightarrow \mathcal{R}_{0}^{+}$which is co-meet-modular and co-join-modular is modular.

¿From the fact that $f$ satisfies co-meet-modularity, i.e. $\forall x, y, z \in X . f(y \sqcap z)-$ $f(x \sqcap z) \geq f(y)-f(x \sqcap y)$, we obtain for $x=a, y=a \sqcup b$ and $z=b$ that $f(b)-$ $f(a \sqcap b)=f(y \sqcap z)-f(x \sqcap z) \geq f(y)-f(x \sqcap y)=f(a \sqcup b)-f(a)$ and hence $f(a \sqcup b)+f(a \sqcap b) \leq f(a)+f(b)$.

Similarly, from the fact that $f$ is co-join-modular, i.e. $\forall x, y, z \in X . f(y \sqcup z)-f(x \sqcup$ $z) \leq f(y)-f(x \sqcup y)$, we obtain for $x=a, y=a \sqcap b$ and $z=b$ that $f(a \sqcup b)+f(a \sqcap b) \geq$ $f(a)+f(b)$. Hence modularity of $f$ follows.

We obtain the following immediate corollary.

Corollary 7 A function on a lattice is a valuation iff it is a join valuation and a meet valuation. A function on a lattice is a co-valuation iff it is a join co-valuation and a meet co-valuation.

The last result considerably strengthens the motivations given of [Sch98] for the fact that semivaluations provide suitable generalizations of valuations from the context of lattices to the context of semilattices. Indeed, Corollary 7 allows one to express a (co-)valuation in terms of two separate semilattice conditions, via the notions of a meet-(co-)valuation and of a join-(co-)valuation.

Finally, we remark that the above results do not hold at the more basic level of modular functions!

¿From the second part of the proof of Proposition 6, we obtain that any function which is both (co)-join-modular and (co)-meet-modular is modular. The converse is not true however in general, but does hold for monotone functions, i.e. functions which are increasing or decreasing, as shown in the first part of the proof. 
The following simple counterexample provides a modular function which is neither (co)-join-modular nor (co)-meet-modular. We leave the straightforward verifications to the reader.

Counterexample: Consider the four point lattice $L=\{x, y, x \sqcap y, x \sqcup y\}$, where $x \neq y$ and let $f$ be a function on $L$ defined by: $f(x)=2, f(y)=8, f(x \sqcap y)=3$ and $f(x \sqcup y)=7$. We remark that $f$ is a modular function which is neither increasing nor decreasing. Moreover, $f$ is neither (co)-join-modular nor (co)-meet-modular.

\section{References}

[Bir84] G. Birkhoff, Lattice Theory, AMS Colloquium Publications 25, Providence, Rhode Island, 1984.

[BS97] M. A. Bukatin, J. S. Scott, Towards computing distances between programs via Scott domains, in: S. Adian, A. Nerode, eds., Logical Foundations of Computer Science, Lecture Notes in Computer Science, 1234, 33-43, Springer, 1997.

[BSh97] M. A. Bukatin, S. Y. Shorina, Partial metrics and Co-continuous Valuations, preprint, Brandeis University, USA.

[CD73] P. Crawley, R. P. Dilworth, Algebraic Theory of Lattices, Prentice-Hall, Inc., 1973.

[Eda94] A. Edalat, Domain Theory and Integration, LICS '94, IEEE Computer Society Press, 1994.

[FSW96] R. Flagg, P. Sünderhauf, K. Wagner, A Logical Approach to Quantitative Domain Theory, Preprint, 1996.

[Hec94] R. Heckmann, Spaces of valuations, in: Proc. 11th Summer Conference on General Topology and Applications. Ann. New York Acad. Sci. 806, 1996, 174 -200 .

[Hec95] R. Heckmann, Lower bag domains, Fundamenta Informaticae 24 (3), 1995, $259-281$.

[Hec96] R. Heckmann, Approximation of Metric Spaces by Partial Metric Spaces, University of Saarland, Germany, 1996.

[JP89] C. Jones, G. Plotkin, A probabilistic powerdomain of evaluations. In: LICS '89, IEEE Computer Society Press, 186 - 195.

[Jon89] C. Jones, Probabilistic Non-determinism, PhD-thesis, University of Edinburgh, 1989. 
[Ko82] R. Kopperman, Lengths on semigroups and groups, Semigroup Forum Vol. 25, 1982, 345 - 360 .

[Kün93] H. P. Künzi, Nonsymmetric topology, in: Proc. Szekszárd Conference, Bolyai Soc. Math. Studies 4, 1993, 303-338.

[KV94] H. P. Künzi, V. Vajner, Weighted quasi-metrics, in: Proc. 8th Summer Conference on General Topology and Applications. Ann. New York Acad. Sci., 728, 1994, 64-77.

[Law95] J. D. Lawson, Valuations on continuous lattices. In: Continuous Lattices and Related Topics (R. E. Hoffman, Ed.), Mathematik Arbeitspapiere 27. Universität Bremen.

[Mat94] S. G. Matthews, Partial metric topology, in: Proc. 8th Summer Conference on General Topology and Applications. Ann. New York Acad. Sci. 728, 1994, 183-197.

[Mat95] S. G. Matthews, An extensional treatment of lazy data flow deadlock, Theoretical Computer Science 151, 195 - 205, 1995.

[O'N97] S. J. O'Neill, Partial metrics, valuations and domain theory. In S. Andima et al., eds., Proc. 11th Summer Conference on General Topology and Applications, Annals New York Academy of Sciences, 806, 304-315, New York, 1997.

[RS96] S. Romaguera, M. Schellekens, Quasi-metric properties of Complexity Spaces, Topology and its Applications, to appear.

[Sch95] M. P. Schellekens, The Smyth Completion: A Common Foundation for Denotational Semantics and Complexity Analysis, in: Proc. MFPS 11, Electronic Notes in Theoretical Computer Science, Vol. I, Elsevier, 1995, 211-232.

[Sch96] M. P. Schellekens, On upper weightable spaces, in: Proc. 11th Summer Conference on General Topology and Applications. Ann. New York Acad. Sci. 806, 1996, 348-363.

[Sch97] M. P. Schellekens, Complexity Spaces: Lifting \& Directedness, Topology Proceedings 22, 403 - 425, 1999.

[Sch98] M. P. Schellekens, On the dual correspondence between partial metrics and generalized (e)valuations in Domain Theory, preprint, 1998.

[Sed96] A. K. Seda, Quasi-metrics and fixed points in computing, Bulletin of the EATCS 60, 1996. 
[Smy91] M. B. Smyth, Totally bounded spaces and compact ordered spaces as domains of computation, in G. M. Reed, A. W. Roscoe and R. F. Wachter, editors, Topology and Category Theory in Computer Science, 207-229, Oxford University Press, 1991.

[Web91] H. Weber, Uniform Lattices I: A generalization of topological Riesz spaces and topological Boolean Rings. Ann. Mat. Pura Appl. 160, 1991, 347 - 370.

National University of Ireland, Cork

Department of Computer Science

Cork

Ireland

E-mail: m.schellekens@cs.ucc.ie 